\title{
Paradise revealed: first-class science rocked by the sound of the waves
}

\author{
GP Amarante-Mendes ${ }^{*, 1,2}$, KR Bortoluci ${ }^{3}$, PT Bozza ${ }^{4}$, R Chammas $^{5}$ and JPB Viola ${ }^{6}$ \\ Cell Death and Differentiation (2010) 17, 1368-1372; doi:10.1038/cdd.2010.66
}

Signaling in Cell Death, Cancer and the Immune System (Signaling 2010) - Angra dos Reis, Rio de Janeiro, Brazil - 8-11 February 2010.

Paradise certainly comes in many different flavors in the mind of each individual. Well, many of these flavors were recently combined at a beach resort south of the city of Rio de Janeiro, Brazil, where the first meeting on 'Signaling in Cell Death, Cancer and the Immune System' was held, creating an incredible atmosphere of intellectuality, joy and friendship. There, first-class scientists shared their most recent work and insights with an enthusiastic group of young scientists and graduate students. Around 200 people gathered at the conference and poster section rooms, the overflowing restaurant or the surroundings of a complex of swimming pools and bars. Seasoning the intense scientific discussions were the spectacular view of the ocean, fine sand beach, coconut water and caipirinhas!

The aim of this meeting was to put together a multidisciplinary scientific program with focus on signaling transduction that could gather scientists from the fields of cell death, tumor biology and immunology. As you will be able to appreciate from the next paragraphs, the results were outstanding.

The meeting started with a symposium that highlighted novel mechanisms operating in cell death, cancer and innate immunity. Seamus Martin (Trinity College Dublin, Ireland) revealed a novel p53-independent, ERK-regulated pathway responsible for the induction of a proapoptotic member of the Bcl-2 family, namely NOXA, important for the chemotherapeutic properties of platinum compounds. Scott Lowe (Howard Hughes Medical Institute and Cold Spring Harbor Laboratory, USA) put forward powerful global genetic approaches based on ex vivo manipulation of stem cells, in particular with libraries of shRNA, followed by transplantation of these cells to syngeneic recipient mice. As a consequence, his team can now create mosaic mouse models to study the role of tumor suppression genes and the cell death machinery in tumor maintenance or regression. Luke O'Neill (Trinity
College Dublin, Ireland) showed that the small $G$ protein Rab39a seems to contain a putative caspase-1 cleavage site at D148. Because the removal of Rab39 from phagolysosomes is essential for its acidification and the knocking down of Rab39a inhibits IL- $1 \beta$ secretion but not TNF- $\alpha$, O'Neill proposed that Rab39a functions as a traffic adapter linking caspase- 1 to IL- $1 \beta$ secretion.

Taking advantage of his innovative mind, Ruslan Medzhitov (Howard Hughes Medical Institute and Yale University School of Medicine, USA) presented the Keynote Lecture offering us his personal view about the formation and maintenance of tissues in multicellular organisms. Anchored in elegant results from his group as well as from others, he called our attention to the existence of a certain level of control of life and death in tissues based on the relative fitness of cells. Interestingly, less fitted or 'loser' cells are only eliminated in the presence of 'winners', suggesting the existence of a mechanism aiming to enhance body fitness, but that still preserves architecture and function of the less robust tissue. Passive competition takes place predominantly (or maybe only) during restrictive situations (stress, or limitations in space, growth factors, nutrients, etc.) and seems to be dependent on cell-intrinsic differences to react to the restrictive environment. A major player identified by Medzhitov and colleagues is p53, expression of which is higher in 'loser' than 'winner' cells. Active competition, however, is likely to be cell extrinsic and may occur at the steady/homeostatic state of a given tissue. 'Winners', in this case, may actively suppress or even kill the 'losers', and the 'losers' may even promote the growth of the 'winners'. Importantly, in any of this situations, a complex set of communication signals appears to control the 'social behavior of cells', aspiring for a healthier organism. Also, at least at the cellular level, the Darwinian 'survival of the fittest' is based not only in competition, but also in cooperation and sacrifice.

\footnotetext{
${ }^{1}$ Departamento de Imunologia, Instituto de Ciências Biomédicas, Universidade de São Paulo, São Paulo, SP, Brazil; ${ }^{2}$ Instituto de Investigação em Imunologia, Instituto Nacional de Ciência e Tecnologia, São Paulo, SP, Brazil; ${ }^{2}$ Departamento de Ciências Biológicas, Universidade Federal de São Paulo - Campus Diadema, Diadema, SP, Brazil; " Laboratório de Imunofarmacologia, Instituto Oswaldo Cruz, Fundação Oswaldo Cruz, Rio de Janeiro, RJ, Brazil; ${ }^{5}$ Faculdade de Medicina, Universidade de São Paulo, São Paulo, SP, Brazil and ${ }^{6}$ Divisão de Biologia Celular, Instituto Nacional de Cancer, Rio de Janeiro, RJ, Brazil

${ }^{*}$ Corresponding author: GP Amarante-Mendes, Departamento de Imunologia, Instituto de Ciências Biomédicas, Universidade de São Paulo, Av. Prof. Lineu Prestes, 1730, Cidade Universitária, 05508-900, São Paulo, SP, Brazil. Tel: + 55113091 7362; Fax: + 55113091 7224; E-mail: gpam @ usp.br
} 


\section{Regulation of Signal Transduction and Gene Expression}

Emer S Ferro (Instituto de Ciências Biomédicas, Universidade de São Paulo, Brazil) showed evidence that peptides similar to those released by the proteasome could keep a biological activity, interfering with intracellular signaling, protein/protein interactions or both, thereby acting as natural regulators of signaling events. High-throughput peptidomics is now being applied in different pathophysiological settings, and soon these data will be correlated with different diseases. Eventually, causal associations may be found, as it has been suggested in the deposition of protein aggregates. Anticipating that, Ferro and colleagues are developing the tools to transform these natural peptides into signaling or supramolecular protein structure disruptors. Anjana Rao (Harvard Medical School, USA) addressed the importance of hydroxymethylation of DNA in regulating gene expression and myeloid tumorigenesis. Rao showed that the expression of TET2, an enzyme that converts 5-methylcytosine to 5 -hydroxymethylcytosine (5-hmC) in DNA, causes loss of 5-methylcytosine in parallel with an increased level of 5-hydroxymethylcytosine in genomic DNA. This activity is abolished by mutations that impair the catalytic activity of the enzyme, as well as by leukemia-associated mutations in residues that have a role in catalysis. Also, she showed that the mutational status of the TET2 locus correlated with the level of 5-hmC in genomic DNA from patients with different myeloid malignancies. Finally, Rao's findings indicate that appropriate hydroxymethylation of DNA is critical for suppressing myeloid tumorigenesis. Sankar Ghosh (Columbia University, USA) presented some exciting results on the regulation of immune and inflammatory responses by NF- $\kappa \mathrm{B}$. Using molecular, biochemical and genetic in vivo strategies, Ghosh's group was able to show that different $I_{\kappa} \mathrm{B}$ isoforms exert specific biological effects by the activation of different individual members of the NF- $\kappa \mathrm{B}$ family, culminating in the regulation of different sets of genes during cell activation. Therefore $\mathrm{NF}-k \mathrm{~B}$ has a central, evolutionarily conserved role in coordinating immune responses, which control many basic physiological processes and may be involved in a variety of severe pathologies, including inflammatory and autoimmune diseases. Carl Ware (La Jolla Institute for Allergy and Immunology, USA) revealed a novel regulation of the noncanonical (p100 and Rel B) NF- $\kappa$ B pathway, which depends on the NF- $\kappa$ B-inducing kinase (NIK). In unstimulated cells, NIK is targeted for proteasomal degradation by the ubiquitin-NIK E3 ligase formed by TRAF2, TRAF3 and c-IAP and, therefore, unavailable for NF- $\kappa \mathrm{B}$ activation. Lymphotoxin- $\beta$ receptor (LT $\beta R$ ) stimulation disrupts the complex TRAF2/TRAF3/C-IAP/NIK, recruiting TRAF2 and TRAF3 to the receptor and targeting them for degradation. Once NIK is released, it associates with IKK $\alpha$ and activates NF- $\kappa \mathrm{B}$.

\section{$\mathrm{Ca}^{2+}$ Mobilization and Signaling}

Patrick Hogan (La Jolla Institute for Allergy and Immunology, USA) discussed the involvement of sensor protein STIM1 and the channel subunit ORAl1 on the regulation of $\mathrm{Ca}^{2+}$ influx in mammalian T cells. To study STIM1-ORAI1 signaling in vitro, Hogan's group have expressed ORAI1 in yeast Saccharomyces cerevisiae, and isolated sealed membrane vesicles carrying ORAI1 from the Golgi compartment to the plasma membrane. They showed by in vitro $\mathrm{Ca}^{2+}$ flux assays that recombinant STIM1 opens wild-type ORAI1 channels, but not channels assembled from the ORAl1 pore mutant E106Q or the ORAI1 SCID mutant R91W. These experiments show that the STIM1/ORAl1 interaction is sufficient to gate recombinant human ORAl1 channels in the absence of other proteins of the human ORAl1 channel complex. Studying intracellular calcium alterations in neurodegenerative and aging processes, Soraya Smaili (Universidade Federal de São Paulo, Brazil) showed compelling evidences that amyloid- $\beta(\mathrm{A} \beta)$ induces $\mathrm{Ca}^{2+}$ mobilization from the endoplasmic reticulum of rat astrocytes, probably through IP3 receptors, which, in turn, causes Bax translocation from the cytosol to the mitochondria. The importance of this event to the development of Alzheimer's disease and other neurodegenerative and aging process was discussed.

\section{Cell Cycle Control}

Steve Reed (The Scripps Research Institute, La Jolla) is dissecting the molecular mechanisms by which quiescent cells reenter the cell cycle, and showed evidence for novel roles of both Cdk2 and Cdc7 in this process. Cyclin E-Cdk2 promotes the accumulation of Cdc7 kinase, which in turn phosphorylates Mcm2, one of the six MCM proteins found in the pre-replication complex. Phosphorylation of $\mathrm{Mcm} 2$ is a required step for its loading onto chromatin and assembly of the pre-replication complex. Cyclin $\mathrm{E}$ is also involved in earlier steps of the assembly of the pre-replication complex, as the recruitment of the subunits of the origin recognition complex (ORC) to DNA. Adriana Hemmerly (Universidade Federal do Rio de Janeiro, Brazil) showed that ORC1 has a double role in cell-cycle control. It does not only participate in the licensing of DNA replication in the nucleus, but also functions as a regulator of centriole reduplication, limiting its duplication to once per cell cycle, thereby helping to maintain genome integrity.

\section{Signaling in Cell Death}

A dazzling structural analysis of DD ('death domain') complexes based on protein crystallography, electron microscopy and biochemistry was presented by Hao Wu (Cornell University, USA). Among other things, Wu showed attractive images showing that Fas and FADD form an oligomeric structure composed of a layer of five Fas DD associated with another layer of five FADD DD, thereby placing the DED domain of FADD in a proper way as to interact with the DED domain of the caspase-8 forming a higher-magnitude oligomer. Wu also showed that the mutations of Fas that is associated with the autoimmune lymphoproliferative syndrome mapped to the interface of these layers and are detrimental to the death-inducing signaling complex formation. The structure of IRAK-1/IRAK-4/MYD88 and its implication to signaling transduction was also discussed during her talk. Sharad Kumar (Centre for Cancer Biology, Australia) talked about the cell death machinery during Drosophila metamorphosis and pointed out that Dronc is required for most developmental cell death but not for midgut 
cell death. Instead, most of the caspase activity in dying midguts is due to decay. Importantly, inhibition of autophagy, but not caspase activity, delay midgut cell death showing for the first time an in vivo caspase-independent form of PCD. Henning Walczak (Imperial College London, UK) put forward a novel modified tandem affinity purification procedure, which increased the specificity of immunoprecipitation, to study the composition of the TNF-receptor signaling complex (TNF-RSC). Walczak showed that two novel proteins are recruited to the complex, HOIL-1 and HOIP. The two associate to form a linear ubiquitin chain assembly complex responsible for assembling linear head-to-tail ubiquitin chains. HOIL-1 recruitment to the TNF-RSC depends on the E3 ligase activity of C-IAP-1/2 but not of TRAF2. Finally, using knocking down strategies, he showed that HOIL-1 and HOIP are important for proper TNF-induced NF- $\kappa \mathrm{B}$ and JNK activation and, therefore, crucial regulators of some TNF-target genes.

\section{Signaling in Cancer}

The perspective of targeting signaling pathways in cancer was addressed in different systems. Sergio Lira (Mount Sinai School of Medicine, USA) revealed the first chemokine receptor implicated in cancer. US28 is a constitutively active viral chemokine receptor encoded by the HHV5 (CMV) that is involved in $\beta$-catenin stabilization and upregulation of c-myc, survivin and cyclin D1 expression. Transgenic mice expressing US28 in intestinal epithelial cells develop colonic and jejunal adenomas and carcinomas. Simultaneous expression of CCL2 and US28 leads to increased frequency of tumors. Work on the physiological roles of the prion protein $(\mathrm{PrPc})$ conducted by Vilma Martins and colleagues (São Paulo Branch of the Ludwig Institute for Cancer Research, Brazil) has been suggesting that PrPc serves as a scaffolding protein, organizing supramolecular complexes on the cell surface of a variety of normal and tumor cells. PrPc seems involved in survival, proliferation and differentiation of cells, including neurons and glia cells. Dissection of PrPc complexes led to the identification of a putative prion receptor, STI1/Hop. Modulation of Hop/PrPc interaction in tumor cells seems a promising strategy to control tumor growth, as shown by preclinical data. Nigel Waterhouse (Peter MacCallum Cancer Center, Australia) aims at sensitizing tumor cells to cytotoxic lymphocytes. In his own previous work, Waterhouse and colleagues showed that primary human NK cells induced apoptosis of cells whose mitochondria were protected by Bcl-2 in a Granzyme-B-dependent manner. Purified Granzyme B could also trigger apoptosis of cells overexpressing Bcl-2. However, the amount of Granzyme B needed to kill these cells was one order of magnitude higher than the amount necessary to kill cells expressing endogenous levels of Bcl-2. Granzyme B triggers cell death through two hierarchically dependent pathways: one which is Bcl-2 inhibitable, mitochondrial dependent and caspase independent, and a second, which is mitochondrial independent and caspase dependent. Granzyme B exerts a pressure for cells to die and experimental data indicated that if following Granzyme B exposure, Bcl-2 activity was inhibited by the small molecule ABT-737, cell death would occur. However, inhibition of $\mathrm{Bcl}-2$ needs to take place within $16 \mathrm{~h}$ after exposure to Granzyme B: a narrow window of opportunity. A challenge for the future will be to translate this elegant concept into therapy. Gerry Melino (MRC, England and Universitá di Roma Tor Vergata, Italy) presented data on a member of the ubiquitin-specific protease (USP) family; USP47, a ligand but not a substrate of $\beta$-Tcrp. Interestingly, both genetic and siRNA-mediated knockdown of USP47 induced accumulation of cdc25a, decreased cell survival and chemosensitized cells to known chemotherapeutic drugs. Taken together, the data suggest USP47 as a novel target for anticancer therapies. As a physician-scientist, Carlos Gil (Instituto Nacional de Câncer, Brazil) commented on the hurdles of translating promising strategies, such as those presented in the meeting to the patient's bedside. Although it seems clear that this will be the century of targeted therapies, we still know little about the diseases we intend to treat. Facing the heterogeneity of cancers at the molecular level, as pointed by Scott Lowe just in the beginning of the meeting, there is simply no reason to insist on a single treatment to different (molecular) diseases, even if the pathologist diagnoses them all as the same disease (at the cellular or tissue level).

\section{Signaling in Inflammation and Innate Immunity}

An intense and productive discussion on the detailed signaling mechanisms in response to inflammation and infection was engaged during the meeting. In an elegant series of imaging and functional studies in whole eosinophils and purified granules, Peter Weller (Harvard Medical School, USA) detailed the signaling mechanisms that regulate the selectivity of human eosinophil secretion. It is known that human eosinophils contain within their granules multiple preformed proteins, including over 30 cytokines and 4 distinctive cationic proteins that may be rapidly mobilized on eosinophil stimulation and contribute to eosinophil-mediated inflammation and immunomodulation. However, the mechanisms that govern the selective release of proteins from within the granules are not well appreciated. Weller showed that granules of human eosinophils express membrane receptors for cytokines/ chemokines, and that these receptors function by triggering signaling pathways within granules to elicit selective secretion of their components. In addition, Weller went on to show that eosinophil granules might function as autonomous organelles outside of eosinophils as ligand-responsive, secretioncompetent structures. Mauro Teixeira (Universidade Federal de Minas Gerais, Brazil) argued about the importance of the selective and timely induction of apoptosis of granulocytes for the resolution of inflammation and showed that cyclic AMP is important in resolving inflammation by cell-specific mechanisms. Although the actions of cAMP in eosinophilic inflammatory resolution are dependent on PKA and target a PI3K/ Akt-dependent NF- $\kappa \mathrm{B}$ survival pathway, cAMP effects on neutrophilic inflammation resolution occur through caspasedependent apoptosis of inflammatory cells by targeting an NF- $\kappa$ B-independent PKA/PI3K/Akt-dependent survival pathway. Rafael Linden (Universidade Federal do Rio de Janeiro, Brazil) addressed the role of the prion protein PrPc in the control of phagocytic and inflammatory responses. Macrophages from PrPc gene knockout mice have higher rates of 
phagocytosis than wild-type macrophages. Moreover, the inflammatory responses involving activation of either TLR2 or TLR4 are tuned by PrPc. Conversely, inflammation leads to induction of PrPc. The existence of these regulatory loops anticipates the involvement of PrPc in autoimmunity, boosting (even more) the interest in the mad cow disease protein. Elvira Saraiva (Universidade Federal do Rio de Janeiro, Brazil) talked about the control of infection by NETosis, a recently described form of cell death that occurs in neutrophils in response to certain parasites. NETosis is characterized by the release of neutrophil extracellular traps (NETs), which is formed by a fibrous network composed of pieces of DNA, histones and granule proteins, such as elastases. Saraiva presented startling pictures and quantitative data showing that Leishmania and other parasites induce the release of and are killed by NETs. Interestingly, disruption of NETs by DNase treatment or by anti-histone antibodies increased parasite survival. Meshes composed of DNA and elastase were evidenced in biopsies of human cutaneous leishmaniasis. Also working on Leishmania, Marcela Lopes (Universidade Federal do Rio de Janeiro, Brazil) showed that L. major infection of B6 mice expressing v-FLIP (a viral inhibitor of caspase-8) in T cells resulted in increased T-cell survival and IL-4 production compared with wild-type mice. Despite the intense Th2 response, which is normally harmful to the mice, B6/v-FLIP mice developed strong resistance to $L$. major infection associated with expansion of myeloidderived suppressor cells capable of producing nitric oxide. Signaling lymphocyte activation molecule (SLAM), a glycoprotein expressed on activated lymphocytes and antigenpresenting cells, has been shown to function as co-stimulatory molecules through their homophilic or heterophilic interactions. Cox Terhorst (Harvard Medical School, USA) expanded the role of SLAM family of receptors to innate immunity by showing that these receptors function as bacterial sensors capable of discriminating Gram-positive from Gram-negative bacteria thereby initiating innate immune signaling. The nodlike receptor (NLR) family constitutes a group of cytoplasmic sensors of pathogen-associated molecular patterns, which trigger signaling cascades that culminate with $\mathrm{NF}-\kappa \mathrm{B}$ or caspase- 1 activation. Dario Zamboni (Faculdade de Medicina de Ribeirão Preto, Universidade de São Paulo, Brazil) presented data that unequivocally show that Coxiella infection does not trigger caspase- 1 activation and consequently may somehow avoid recognition by NLRs. Moreover, by performing co-infections with Legionella, Zamboni went on to show that Coxiella is capable of inhibiting caspase- 1 activation by Legionella-infected cells, giving support to the hypothesis that Coxiella is in fact able to subvert activation of caspase-1 and the inflammasome, possibly aiming to establish a replicative vacuole that enables intracellular microbial multiplication. As to malaria pathogenesis, the signaling determinants of malaria-induced cytokine storm that leads to fever and sepsis are poorly understood. Douglas Golembock (University of Massachusetts Medical School, USA) presented novel findings revealing the importance of a synergistic effect of $P$. falciparum DNA and hemozoin as the cause of fever in malaria. Golembock clearly showed that although hemozoin does not directly triggers TLR9 activation, it is crucial in the intracellular trafficking of $P$. falciparum DNA to trigger TLR9/
MyD88-dependent and -independent signaling pathways for cytokine production. The TLR9/MyD88 independent activation was shown to occur through NALP-3 and ASC. These findings may open new avenues for the therapeutic intervention in malaria. Miguel Soares (Instituto Gulbenkian de Ciência, Portugal) discussed novel molecular aspects involved in host tolerance to infection. Soares presented solid evidence that free heme is released during severe infection including malaria and polymicrobial sepsis and functions as a common pathological mechanism that impairs host tolerance to infection, leading to cell death and tissue damage. He also discussed the role of stress-responsive genes, in particular the key role played by $\mathrm{HO}-1$, in limiting the deleterious effects of free heme preventing tissue damage and promoting host survival. Ricardo Gazzinelli (Universidade Federal de Minas Gerais, Brazil) revealed a critical role for UNC93B1, a multiple membrane-spanning endoplasmic reticulum protein, on the control of Toxoplasma infection. Interestingly, Gazzinelli presented convincing findings that although UNC93B1deficient mice mount a typical proinflammatory response with abundant IFN- $\gamma$, the animals were unable to control parasite replication. Therefore, UNC93B1 seems to control Toxoplasma infection through mechanisms independent of TLR function and dependent of the trafficking of intracellular parasite-containing vesicles. Novel survival strategies and host/defense evasion mechanisms triggered by Brucella spp. and $M$. tuberculosis (Mtb) were also discussed. The innate immune recognition and signaling triggered by Brucella spp. infection are particularly very poorly understood. Sérgio Costa Oliveira (Universidade Federal de Minas Gerais, Brazil) provided a solid body of evidences to conclude that $B$. abortus and its DNA has the ability to induce type I IFN synthesis, independently of TLR2, TLR4, TLR9 or TRIF, but dependent on MyD88 and IRF-3. Interestingly, splenocytes from IFN- $\alpha \beta$ R-deficient mice showed a significant increase in IFN- $\gamma$ and NO level when cultured with $B$. abortus and a lower apoptotic index when compared with wild-type mice, suggesting that the $B$. abortus-mediated production of type I IFN is one of the host/defense evasion mechanisms triggered by this pathogen. By using a combination of genome-wide microarray and immunohistological analysis of caseous human TB granulomas, David Russell (Cornell University, USA) provided biochemical and molecular evidence that the transition of the human tuberculosis granuloma to caseation is promoted by pathogen-mediated deregulation of lipid metabolism leading to lipid accumulation and foamy macrophage formation. This mechanism involves TLR2 and MARCO-dependent signaling and implicates the innate immune system in disease progression. Jean Pieters (University of Basel, Switzerland) discussed the mechanisms involved in the regulation of Mtb and T-cell survival through coronin-1, a leukocyte-specific regulator of $\mathrm{Ca}^{2+}$-dependent signaling. Pieters showed that on infection, coronin-1 is actively recruited to mycobacterial phagosomes. Although coronin-1 is dispensable for all F-actin-mediated functions, including phagocytosis, it is required for the activation of the $\mathrm{Ca}^{2+}$-dependent phosphatase calcineurin processes in macrophages, revealing a function for calcineurin in the regulation of phagosom/lysosome fusion upon mycobacterial infection. 


\section{Signaling in Adaptive Immunity}

Th17 cells are considered the most recent double-edged sword of the immune system. Vijay Kuchroo (Harvard Medical School, USA) and Dan Littman (HHMI and New York University, USA) gave remarkable overviews about the molecular mechanisms that control Th17 cell differentiation. On the basis of expression profiling and systems biology approaches, Kuchroo showed that the expression of orphan nuclear receptor ROR $\gamma \mathrm{T}$, a key transcription factor regulating Th17 cell development described by Littman's group, is initiated early on TGF- $\beta 1$ and IL- 6 stimulation. This induction phase is followed by upregulation in the IL-21 expression, a key factor for Th17 cell amplification. Finally, IL-23R expression and signaling lead to Th17 phenotype stabilization. By using chromatin immunoprecipitation followed by highthroughput sequencing (ChIP-Seq), Littman showed that other nuclear factors, for example STAT3 and IRF-4, collaborate with ROR $\gamma$ t to regulate transcription and the generation of IL-17-producing cells. Beneficial and pathological roles of Th17 cells were also presented in this section. Illustrated by wonderful scanning electron microscopy pictures, Littman showed that the accumulation of Th17 cells in murine intestinal mucosa colonized by segmented filamentous bacteria protects mice from overgrowth of pathogenic intestinal bacteria. Kuchroo highlighted another feature of Th17 by showing their cooperation with $B$ cells leading to B-cell proliferation, antibodies class switch to $\lg G 1, \lg G 2 a$ and IgG3 isotypes, and germinal center formation. The pathologic role of Th17 cells was also shown by Kuchroo where in vitro derived Th17 cells from naive TCR transgenic mice with specificity for myelin antigens induce a more severe EAE when compared with Th1 cells of the same specificity. Alexander Rudensky (HHMI and University of Washington, USA) discussed the features of regulatory $T$ cells (Tregs) responsible for the immune homeostasis and for the suppressing overexuberant immune responses. He focused on the role of conserved non-coding DNA sequence (CNS) elements at the Foxp3 locus for the determination of Treg fate. Rudensky's group is addressing the particular requirement of distinct Foxp3 CNS elements for the control of thymus or peripheral Treg subpopulations composition, size and maintenance. Focusing on $\mathrm{CD}^{+} \mathrm{T}$ cells, Mauricio Rodrigues (Universidade Federal de São Paulo, Brazil) proposed an efficient vaccine strategy for experimental Chagas' disease by using a replication-deficient human adenovirus type 5 expressing the $T$. cruzi immunodominant CD8 epitopes. Specific $\mathrm{CD}^{+} \mathrm{T}$ cells expanded following adenoviral vaccination, acquiring a typical T-effector phenotype and cytotoxic activity. Moreover, these cells drastically downmodulated the expression of the apoptotic signaling receptor CD95 (Fas) induced by pathogen infection. Thus, in addition to the induction of effector immune cells before the pathogen encounter, the adenoviral vaccination seem to reprogram pathogen-specific $\mathrm{CD}^{+}{ }^{+} \mathrm{T}$ cells expanded after an infectious challenge. In an enthusiastic talk, Hilde Cheroutre (La Jolla Institute for Allergy and Immunology, USA) showed that the gut environment selects for the 'fittest' immune cells through TL-mediated signals from epithelial cells. TL induces apoptosis in activated CD $8 \alpha \beta$ T cells, but it is inhibited by the expression of activation-induced CD8 $\alpha \alpha$ in these cells. Interestingly, TGF- $\beta$, abundant in the gut, is required for the conversion of CD4 T cells into CD8 $\alpha \alpha C D 4 T$ cells that, in their turn, is capable to acquire a CD8 cytotoxic phenotype. Mitchell Kronenberg (La Jolla Institute for Allergy and Immunology, USA) used different experimental systems to investigate the role of TNF receptor family HVEM in T-cell responses. Using a model of colitis induced by transfer of $\mathrm{CD}^{+} \mathrm{CD} 45 \mathrm{RB}$ high $T$ cells into rag- deficient mice, Kronenberg and colleagues verified that the absence of HVEM in radio-resistant cells, probably the intestinal epithelial cells, resulted in accelerated intestinal inflammation. Administration of agonistic anti-BTLA antibodies prevented the accelerated colitis, highlighting the importance of the HVEM-BTLA interaction to the control of intestinal inflammation. An apparent opposing effect of HVEM was seen in mice infected with Citrobacter rodentium, since HVEM deficiency resulted in impaired bacterial clearance and increased susceptibility to infection.

\section{Closing Remarks}

The meeting was enthusiastically wrapped up by Douglas Green (St. Jude Children's Research Hospital, USA), who pointed out evolutionary aspects as well as the importance of mitochondrial events in the control of life and death of vertebrate cells. He reviewed and discussed the fundamental mechanisms controlling the mitochondrial outer membrane permeabilization (MOMP), the critical step for effector caspase activation and apoptosis, and also for caspaseindependent cell death. MOMP is controlled by pro- and antiapoptotic members of the Bcl-2 family. Bax and Bak are the proapoptotic effectors and Bcl-2, Bcl-xL and MCL-1 are the antiapoptotic proteins that regulate the so-called $\mathrm{BH} 3$-only members of this family (Bid, Bim, Bad, Noxa, Puma and so on). Green argued in favor of the existence of both the 'neutralization' and the 'direct activator/derepressor' models of function of $\mathrm{Bcl}-2$ family proteins and drew attention to the fact that each one leads to different outcome, with particular implications for the development of therapeutic interventions. Last, but not least, he revisited the concept of 'commitment point' he proposed some years ago, reemphasizing the consequence of MOMP to mitochondrial dynamics and bioenergetics as a mechanism to ensure death of a cell even in the absence of caspase activation.

\section{Conflict of interest}

The authors declare no conflict of interest.

Acknowledgements. We thank our speakers and attendees for making this meeting a successful scientific and interactive event. We apologize to those speakers whose work is not discussed owing to space limitations. We are indebted to CNPq, FAPESP, FAPERJ, CAPES, FIOCRUZ, Instituto de Investigação em Imunologia (INCT-iii), Instituto Nacional de Ciência e Tecnologia de Vacinas (INCTV), Instituto Israelita de Ensino e Pesquisa Albert Einstein, BD (Becton Dickinson and Company) Brasil, Sociedade Brasileira de Imunologia and Kyowa Kirin California (KKC) for the financial support. 